\title{
PHOTOMETRIC PROCEDURE FOR QUANTITATIVE ANALYSIS OF AFLATOXIN B1 IN PEANUTS BY THIN- LAYER CHROMATOGRAPHY USING CHARGE COUPLED DEVICE DETECTOR
}

\author{
Michele Hoeltz*, Juliane Elisa Welke e Isa Beatriz Noll \\ Instituto de Ciência e Tecnologia de Alimentos, Universidade Federal do Rio Grande do Sul, Av. Bento Gonçalves, 9500 , \\ 91570-901 Porto Alegre - RS, Brasil \\ Horacio Alberto Dottori \\ Instituto de Física, Universidade Federal do Rio Grande do Sul, Av. Bento Gonçalves, 9500, 91570-901 Porto Alegre - RS, Brasil
}

Recebido em 12/11/08; aceito em 1/7/09; publicado na web em 25/11/09

\begin{abstract}
A photometric procedure was developed for determination of aflatoxin $B_{1}$ in peanuts by TLC-CCD technique. The quantification and detection limit were $1.2 \mu \mathrm{g} \mathrm{kg}^{-1}$ and $0.4 \mathrm{ng}$ per spot, respectively, with mean recovery of $98 \%$. The CCD camera is sufficiently sensitive to detect small changes in spots fluorescence intensity and the results for performance confirmed the efficiency of the method. Another important property of CCD detector is its linearity for a wide range of luminous stimulus determined by analysis of five-point calibration curves using the intensity of AFB1 fluorescence versus AFB1 concentration ( 0.8 to $4.8 \mathrm{ng}$ per spot). The method was applied to the analysis of thirty nine peanut samples and aflatoxin $\mathrm{B}_{1}$ levels ranged from 16 to $115 \mu \mathrm{g} \mathrm{kg}$. The TLC-CCD and the photometric procedure developed in this study demonstrated to be a simple and efficient tool for quantitative analyses of AFB1 in peanut samples.
\end{abstract}

Keywords: aflatoxin B1; photometric procedure; TLC-CCD.

\section{INTRODUCTION}

Aflatoxins $\mathrm{B}_{1}, \mathrm{~B}_{2}, \mathrm{G}_{1}$ and $\mathrm{G}_{2}$ are difuranocoumarin derivatives produced by a polyketide pathway by many strains of Aspergillus flavus Link. ex Fries, A. parasiticus Speare and A. nomius Kurtzman, B.W. ${ }^{1}$ These mycotoxins have been found in different food such as corn, peanut, rice and walnut., ${ }^{2,3}$

Crops in tropical and subtropical areas are more susceptible to contamination than those in temperate regions, since the high humidity and temperature in these areas provide optimal conditions for toxin formation. Poor harvesting practices, improper storage and less than optimal conditions during transportation, marketing and processing can also contribute to fungal growth and increase the risk of mycotoxin production. ${ }^{4}$ Peanuts are known to be a major substrate for aflatoxigenic fungal species. ${ }^{5}$

It is most unlikely that commodities will contain aflatoxins $\mathrm{B}_{2}$, $\mathrm{G}_{1}$ and $\mathrm{G}_{2}$ and not aflatoxin $\mathrm{B}_{1}{ }^{6}$, and the concentration of the sum of the aflatoxins $B_{2}, G_{1}$ and $G_{2}$ is generally less than the concentration of aflatoxin $\mathrm{B} 1$ alone. For that reason, analysis of one target component (AFB1) seems to be efficient, sufficient and more practical. ${ }^{6}$

Aflatoxin $\mathrm{B}_{1}$ (AFB1) (Figure 1) is the most toxic form of mycotoxin for mammals and presents hepatotoxic, teratogenic and mutagenic properties, causing damage such as toxic hepatitis, hemorrhage, edema, immunosuppression and hepatic carcinoma. ${ }^{7}$ The sum of aflatoxins $B_{1}, B_{2}, G_{1}$ and $G_{2}$ has been classified as class 1 carcinogenicity for humans by the International Agency for Research on Cancer. ${ }^{8}$

Contamination levels vary worldwide. Craufurd et al. ${ }^{9}$ in a study about aflatoxin on peanut in Niger, found values ranged from 34 to $208 \mu \mathrm{g} \mathrm{kg}^{-1}$. Wang and $\mathrm{Liu}^{10}$ analyzing the contamination of aflatoxins in different kinds of foods in China, found highest level of $28.39 \mu \mathrm{g} \mathrm{kg}^{-1}$ in peanuts. Sulaiman et al. ${ }^{2}$ studying the occurrence of aflatoxins in raw shelled peanut samples from three districts of Perak, Malaysia, found the range of incidence of the AFB1 from 0.85 to $547.5 \mu \mathrm{g} \mathrm{kg}^{-1}$. In a review about mycotoxin research in Brazil from years 1991 to 2000, Rodriguez-Amaya and Sabino ${ }^{11}$

*e-mail: michelehoeltz@yahoo.com.br reported aflatoxins contamination in peanuts and peanut products from 1 to $13000 \mu \mathrm{g} \mathrm{kg}^{-1}$.<smiles>COC1=CC2=C(C3C=COC13)C1OC(=O)C3=C(CCC3=O)C21</smiles>

Figure 1. Molecular structure of aflatoxin B1 (AFB1)

In general the aflatoxins regulation varies between different countries with limits from 0 to $35 \mu \mathrm{g} \mathrm{kg}^{-1}$. In European Union the maximum level allowed for AFB1 in food is $2 \mu \mathrm{g} \mathrm{kg}^{-1}$ and for the sum of aflatoxins is $4 \mu \mathrm{g} \mathrm{kg}^{-1} \cdot{ }^{12}$ Brazilian regulatory limit in peanuts is $20 \mu \mathrm{g} \mathrm{kg}^{-1}$ for the sum of the concentrations of aflatoxins $B_{1}, G_{1}, B_{2}$ and $G_{2} \cdot{ }^{13}$

Several methods for the aflatoxins determination in various samples have been already developed and reported in the literature. The more common methods used for aflatoxins determination step include thin-layer chromatography (TLC) and high performance liquid chromatography (HPLC) with various detection systems. ${ }^{5,714}$ HPLC is used worldwide because it offers good sensitivity and precision, coupled with ease of automation. ${ }^{15,}{ }^{16}$ However, HPLC is expensive in initial capital investment and requires skilled and experienced staff to operate and maintain equipment. ${ }^{17,}{ }^{18}$ By contrast, TLC is a simple and robust technique, ${ }^{19}$ which is relatively inexpensive to establish in a testing laboratory, and most laboratories in developing countries have considerable expertise and experience with it. ${ }^{20}$ The major problem in TLC method is the quantification that usually is done by visual comparison, however other techniques can be employed to improve the analytical performance, such as densitometry. ${ }^{21}$

Charge-coupled devices (CCDs) are two-dimensional detectors containing an array of sensors that can image an area in fraction of seconds or real time.22 The output from each sensor pixel on the CCD is a voltage, which is proportional to the intensity of light falling on 
the sensor and the exposure time. These series of voltages are digitized and transferred to a computer for storage and data processing. ${ }^{22}$

By coupling CCD detection with TLC, the entire chromatographic plate can be imaged in a single exposure yielding rapid quantification in shorter analysis time than of slit scanning densitometers. ${ }^{23} \mathrm{CCD}$ detectors have demonstrated extremely low dark current and read noise characteristics, high sensitivity and excellent linearity. These features have made the CCD an excellent detector for many imaging applications in chemical analysis, such as fluorescence detection. ${ }^{19,20}$

Image $J$ is one of the many image processing tools available and is required for the analysis of images generated by the CCD camera and to carry out the necessary operations. It is a versatile software, which offers a wide array of functionalities that can be customized to meet specific requirements. ${ }^{22}$ The package is freely available (http:// rsbweb.nih.gov/ij/) and very appropriate for the current application..$^{23}$

The aim of this study was to develop a photometric procedure for determination of aflatoxin $B_{1}$ in peanuts by TLC through the fluorescence images from UV lamp recorded by a CCD camera.

\section{EXPERIMENTAL}

\section{Samples}

A total of 39 samples of peanuts ready for consumption were analyzed for determination of AFB1. Several brands were collected at random at supermarkets and rural fairs in different regions of the States of Rio Grande do Sul, Brazil.

\section{Aflatoxin B1 standard solution}

The concentration and purity of aflatoxin standard were determined by the technique of the Association of Official Analytical Chemists. ${ }^{24}$ A stock standard solution of AFB1 was prepared by dissolving $1 \mathrm{mg}$ of pure crystalline AFB1 (Sigma-Aldrich, Steinheim, Germany) in toluene:acetonitrile $(9: 1, \mathrm{v} / \mathrm{v})$ at concentration of $80 \mu \mathrm{g}$ $\mathrm{mL}^{-1}$. The concentration of the AFB1 stock solution was determined by measuring the UV absorbance at $348 \mathrm{~nm}$ and calculated by using the molar extinction coefficient $\varepsilon$ of 19300 . The concentration of working standard solution in toluene: acetronitrile $(9: 1, \mathrm{v} / \mathrm{v})$ was 0.8 $\mu \mathrm{g} \mathrm{mL} \mathrm{m}^{-1}{ }^{25}$ The standard and working solutions were kept frozen $(-18$ $\left.{ }^{\circ} \mathrm{C}\right)$. The purity of aflatoxin B1 was $92 \%$.

\section{Aflatoxin $B_{1}$ extraction}

The samples weighing approximately $1 \mathrm{~kg}$ were ground and homogenized and a subsample of $50 \mathrm{~g}$ was removed for analysis in duplicate.

AFB1 was determined according to the method described by Soares and Rodriguez-Amaya. ${ }^{26}$ Firstly, $50 \mathrm{~g}$ of each sample was extracted with $270 \mathrm{~mL}$ methanol and $30 \mathrm{~mL} \mathrm{4 \%} \mathrm{(w/v)} \mathrm{potassium}$ chloride. Samples were blended at high speed (Blender, Walita, $550 \mathrm{~W}$ ) for $5 \mathrm{~min}$ and filtered, $150 \mathrm{~mL}$ of the filtrate was collected. After that, $150 \mathrm{~mL} 10 \%$ (w/v) cooper sulfate and $5 \mathrm{~g}$ diatomaceous earth were added, followed by moderate stirring and filtration. 150 $\mathrm{mL}$ of the filtrate was transferred to a separation funnel with 150 $\mathrm{mL}$ of distilled water, and AFB1 was extracted twice with $10 \mathrm{~mL}$ chloroform. The chloroform extracts were colleted and submitted to solvent evaporation in water bath at $60^{\circ} \mathrm{C}$.

\section{Chromatography}

The extracts were resuspended in $100 \mu \mathrm{L}$ chloroform and submitted to thin-layer chromatography (TLC). Three, six and nine microliter ali- quots of sample extract and two, four and six microliter aliquots of AFB1 standard solution $\left(0.8 \mu \mathrm{g} \mathrm{mL}^{-1}\right)$ were spotted $1 \mathrm{~cm}$ apart on precoated silica gel plates $\left(10 \mathrm{x} 10 \mathrm{~cm}^{2}\right)$ (Merck, Germany). The spots were dried, and the plates developed in a saturated chamber with chloroform:acetone (99:1, v/v). Aflatoxins spots were observed under long-wave ultraviolet light $(\lambda=366 \mathrm{~nm})$ and identified by comparison with AFB1 standard. Confirmatory tests for AFB1 were carried out using trifluoroacetic acid. ${ }^{27}$

\section{CCD imaging system}

Mycotoxins naturally fluorescent, separated on a chromatographic plate can be quantitatively analyzed through a photometric procedure that compares the sample spots and the standard solution spots in different concentrations.

The way to carried out this evaluation can be defined as a photometric procedure. In this case, AFB1 fluorescence intensities from UV lamp were recorded by a CCD camera (Sony, Tokyo, Japan).

The TLC plate was positioned in system and CCD camera was aligned for optimal pixel resolution of CCD images. Five images were taken in each experiment and were analyzed using package ImageJ (Image Processing and Analysis in Java, http://rsbweb.nih.gov/ij/). ${ }^{28}$

\section{Photometric procedure}

A photometer was design to ensure that every area element on the plate will get the same number of photons, within desired errors. One important concept is that the light source, the detector and the plate be on the same axis, as shown in Figure 2. The illumination uniformity on a square area of size 1 can be achieved by an optical device or by the geometric distribution of the components previously described, by controlling the distance $\mathrm{L}$ between the source and the chromatographic plate. We choose the geometric design. Furthermore, the light sources and the CCD sensor were set on the same plane, perpendicular to the set axis of symmetry, and consequently parallel to plate plane. As an example, a uniformity of $1-2 \%$ can be reached in the illumination of an area of $10 \times 10 \mathrm{~cm}^{2}$ by distributing $4 \mathrm{UV}$ lamps of $17 \mathrm{~cm}$ around the entrance of the CCD camera, putting them at $100 \mathrm{~cm}$ from the putative area of the chromatographic plate. Appropriate distribution of deckers help to avoid spurious light resulting from rays reflected on the instrument walls. a) Lateral view

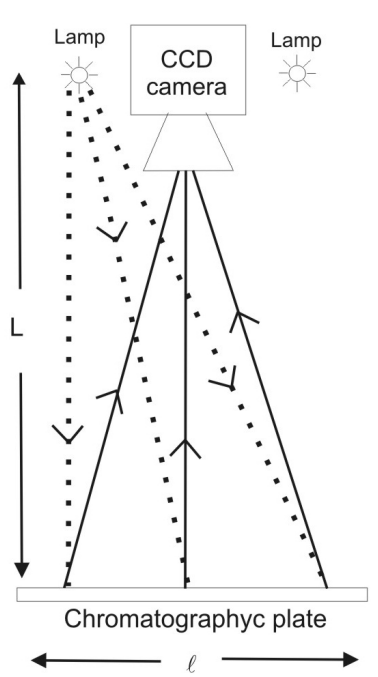

b) Frontal view

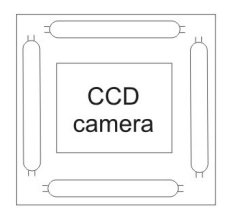

Figure 2. (a) Lateral view of photometer design with perspective of light and (b) frontal view of photometer design showing the location of lamps 


\section{Processing the CCD images}

Two different software can be used to analyze the CCD images: ImageJ (http://rsbweb.nih.gov/ij/) ${ }^{28}$ and IRAF (http://iraf.noao.edu/). ${ }^{29}$ Image $J$ is widely used in medicine ${ }^{30,31}$ and IRAF (Image Reduction Astronomical Facility) is practically the universal package for photometric reduction in Astronomy. ${ }^{32}$ The simplest way to measure the spots brightness is with the so called diaphragm photometry. In that mode one choose the diaphragm size and form (we adopted circular diaphragms due to the spots symmetry). Embracing a spot with this diaphragm one get the total number of pixel elements, the pixel mean brightness, the total brightness and its mean deviation. To discount the background, several backgrounds around every spot were measures with the same diaphragm.

\section{Performance of the analytical method}

The performance of the proposed quantitative method was evaluated based on the following parameters: linearity, recovery percentage, precision, limit of detection (LOD) and limit of quantification (LOQ).

The sensitivity of the TLC-CCD technique was evaluated by determining LOD for AFB1. Decreasing concentrations of AFB1 standard solution (1.0, 0.8, 0.6, 0.4 and $0.2 \mathrm{ng}$ per spot) were analyzed and the LOD was derived from the AFB1 standard fluorescence with the lowest detectable signals. Linearity was determined by analysis of five-point calibration curves using the intensity of AFB1 fluorescence versus AFB1 concentration. The precision was evaluated by taking five successive chromatographic plate images by CCD camera of ten plates containing $0.8,1.6,2.4$, 3.2 and $4.8 \mathrm{ng}$ per spot of standard solution. The fluorescence of each spot was measured three times. The repeatability of AFB1 extraction method and accuracy of TLC-CCD technique were assessed by measuring the percent recovery of uncontaminated samples spiked with 16,20 and $32 \mu \mathrm{g} \mathrm{kg}^{-1}$ of AFB1 in triplicate. The samples were spiked with the desired amount of standard solution at different points on the center top of the mass (50 g) and kept to dry naturally overnight. Within-day precision was determined by analyzing 4 replicates of spiked peanuts sample, and between-day precision was determined by analyzing 5 replicates during 5 days consecutives. The precision of the method was calculated from the relative standard deviation (RSD). The LOQ was obtained by spiking samples with decreasing concentrations of AFB1 standard solution $\left(2.4,2.0,1.6,1.2\right.$ and $\left.0.8 \mu \mathrm{g} \mathrm{kg}^{-1}\right)$ being considered the least amount of AFB1 in samples that can be quantitatively determined with accuracy and precision.

\section{RESULTS AND DISCUSSION}

A chromatogram can be generated from fluorescent spots which correspond to different AFB1 levels by ImageJ software, showing the ability of the CCD camera to take analytical information (Figure 3). Each peak corresponds to a concentration of standard solution (2.4, 4.8, 7.2, 9.6 and $12 \mathrm{ng}$ ) spotted on chromatographic plate. The CCD camera is sufficiently sensitive to detect changes in spots fluorescence intensity caused by small differences in mycotoxin concentration under a homogeneous illumination from a UV light source. The change in fluorescence signal intensity corresponds to the different concentrations of AFB1 standard.

The photometric procedure developed in this study is based in a CCD camera. The light beam is composed by ionizing and non-ionizing photons. Ionizing photons incident on the spots will produce fluorescence, that is, the photons living the spots will have lower energy/frequency than the incident ones. The lower frequency photons will be registered by the CCD camera. The ionizing photons that strike outside the spots will be reflected with the same frequency of incidence. Since they are UV photons outside the CCD sensibility range, they will be not registered by the sensor. The non-ionizing photons will be similarly reflected by the whole chromatographic plate, that is, by the spots and by the adjacent areas. Within this spectrum, the photons within the CCD range of sensibility will be detected by the device inside and outside the spots. That will constitute the background light, undesirably contributing to the spots brightness. This subsidiary brightness has to be discounted from the spot by measuring nearby areas to them. One caveat of this procedure step would be the lack of uniformity of the light beam focusing on the chromatographic plate. That seems to be the case of the photometer used by Zhang and $\operatorname{Lin}^{19}$ where the light focus on the chromatographic plate clearly is not uniformly distributed. In the study of these authors, the number of ionizing photons falling on one spot at the plate center is $60 \%$ larger than those incidents at the border. In cases like that part of the spots brightness difference may be due to the non homogeneity of the light beam, provided that the number of in falling ionizing photons on the less illuminated spot is not enough to excite the whole mycotoxin sample. In that case, a mathematical procedure must be done to correct the background differences between the center and the border.

a)
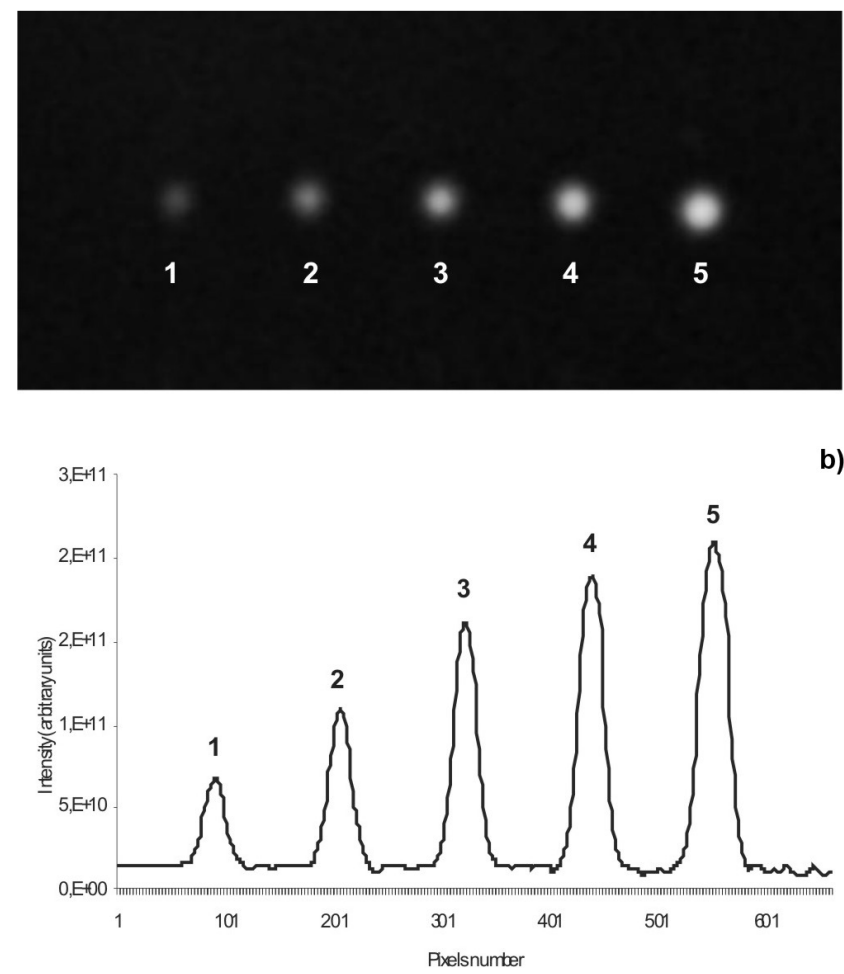

Figure 3. (a) Image of chromatographic plate obtained by the CCD camera with (1) 2.4, (2) 4.8, (3) 7.2, (4) 9.6 and (5) 12 ng of aflatoxin $B_{1}$ standard solution and (b) chromatogram obtained from chromatographic plate image by CCD camera with the same concentrations of aflatoxin B1

A uniform illumination of the spots was achieved, in this study, by an adequate design of the photometer (Figure 2), the device mounted to carry out the photometric procedure.

The effectivity and selectivity of TLC-CCD method can be seen in Figure 4. Peaks 1, 2 and 3 of the chromatogram correspond to three, six and nine microliter aliquots of sample extract while peaks 4 and 5 correspond to two and four microliter aliquots of AFB1 standard solution $\left(0.8 \mu \mathrm{g} \mathrm{mL}^{-1}\right)$. All interferents from the sample are in the baseline of the chromatogram (gray line), not compromising the image analysis. 


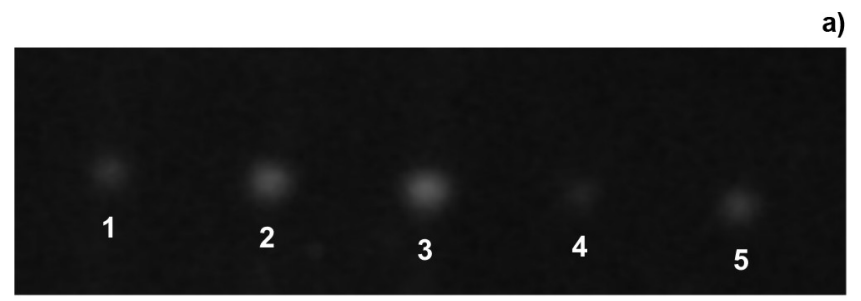

b)

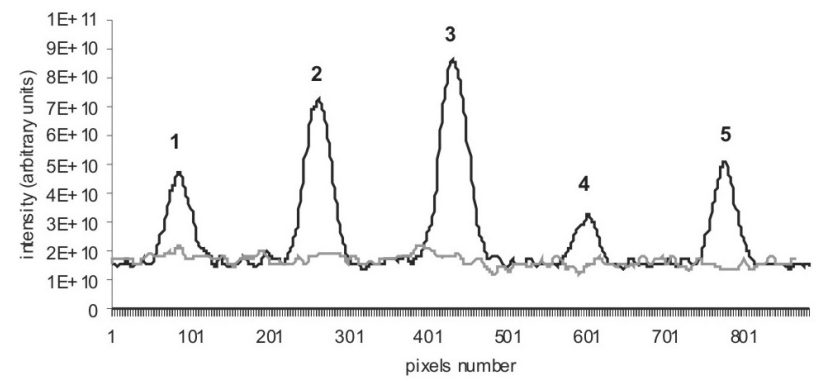

Figure 4. (a) Image of chromatographic plate obtained by the CCD camera with (1) three, (2) six, (3) nine microliters aliquots of sample extract, (4) two and (5) four microliter aliquots of aflatoxin B1 standard solution $(0.8 \mu \mathrm{g} \mathrm{mL}$ $\left.{ }^{1}\right)$; (b) chromatogram obtained from chromatographic plate image by $C C D$ camera with the same concentrations of sample extracts and aflatoxin B1 standard solution showing the interferents from the sample in the baseline of the chromatogram (gray line)

The LOD was $0.4 \mathrm{ng}$ per spot and the LOQ was $1.2 \mu \mathrm{g} \mathrm{kg}^{-1}$. Linearity was determined by analyzing five-point calibration curves within the concentration of $0.8,1.6,2.4,3.2$ and $4.8 \mathrm{ng}$ per spot. The linear regression coefficient of standard solution curve $(y=40255 x$ - 1894.8) was 0.998.

Recovery rates of AFB 1 obtained by spiking peanut samples with 16,20 and $32 \mu \mathrm{g} \mathrm{kg}^{-1}$ in triplicate were 94, 97 and $102 \%$, respectively, and the relative standard deviation (RSD) for repeatability was 4.2, 4.0 and 5.6, respectively. The results of the within and between-day precision of the assay (Table 1) show that the method has good precision, with RSDs $\leq 5.2$.

Table 1. Precision of the extraction method and TLC-CCD technique for determination of AFB1 in peanut samples

\begin{tabular}{lcccccc}
\hline & \multicolumn{3}{c}{ Within-day } & \multicolumn{3}{c}{ Between-day } \\
\hline AFB1 spiked & Recovery & Recovery & RSD & Recovery & Recovery & RSD $^{\text {a }}$ \\
$\left(\mu \mathrm{g} \mathrm{kg}^{-1}\right)$ & $(\mu \mathrm{g} \mathrm{kg}-1)$ & $\%$ & $(\%)$ & $(\mu \mathrm{g} \mathrm{kg}-1)$ & $\%$ & $(\%)$ \\
16 & 15.2 & 95.0 & 4.1 & 15.6 & 97.5 & 4.9 \\
20 & 19.2 & 96.0 & 3.8 & 19 & 95.0 & 3.9 \\
32 & 30.6 & 95.6 & 5.2 & 30.1 & 94.1 & 4.5 \\
\hline
\end{tabular}

${ }^{\mathrm{a}} \mathrm{RSD}=$ Relative standard deviation

The applicability of this method was tested analyzing 39 peanut samples and the Table 2 shows the levels of AFB 1 found. Of the 39 samples analyzed, AFB1 was not detected in $84.6 \%$ of them and $15.4 \%$ showed levels from 16 to $115 \mu \mathrm{g} \mathrm{kg}$.

The high levels of AFB1 contamination in peanuts marketed in southern Brazil are in disagreement with the current Brazilian regulation exceeding the tolerance level of $20 \mu \mathrm{g} \mathrm{kg}^{-1}$ and become important because the toxic effects of aflatoxins represent a severe health risk for consumers and also a risk factor for the economy since the product does not satisfy the requirements for export.
Table 2. AFB1 levels in peanut samples marketed in south of Brazil determined by TLC-CCD technique in duplicate

\begin{tabular}{lcc}
\hline Sample no. & AFB1 $\left(\mu \mathrm{g} \mathrm{kg}^{-1}\right)$ & RSD $^{\mathrm{a}}(\%)$ \\
\hline 1 & 38 & 7.4 \\
2 & 45 & 12.5 \\
3 & 29 & 3.6 \\
4 & 115 & 1.3 \\
5 & 65 & 4.3 \\
6 & 16 & 8.8 \\
\hline
\end{tabular}

${ }^{\mathrm{a}} \mathrm{RSD}=$ Relative standard deviation

Nakai et al. ${ }^{7}$ used TLC to determine the occurrence of aflatoxins in stored peanut samples from State of São Paulo, Brazil, found mean levels of AFB1 from 7.0 to $116 \mu \mathrm{g} \mathrm{kg}^{-1}$ and the detection limit was $2 \mu \mathrm{g} \mathrm{kg}^{-1}$.

Sabino et al..$^{33}$ researching about the occurrence of aflatoxins in peanuts and peanut products consumed in the State of São Paulo, Brazil, found the maximum level of contamination of $536 \mu \mathrm{g} \mathrm{kg}^{-1}$, using TLC technique by visual comparison with the quantification limit of $5 \mu \mathrm{g} \mathrm{kg}^{-1}$.

Usually, the detection limits achieved for the determination of AFB1 in different samples by TLC technique are higher than that found in this study ( $0.4 \mathrm{ng}$ per spot). ${ }^{7,33}$ This probably occurs because the TLC techniques for quantification usually use the visual comparison of fluorescent spots of the samples with fluorescent spots of the standard solution in different concentrations and it is known that the sensitivity of the human eye can not be compared to a photographic image as proposed in this study. Furthermore, the ability to view differs from person to person and results may differ when analyzed by different analysts.

Using TLC and densitometry for determination of AFB1, Prado et al. ${ }^{21}$ obtained mean recoveries of $93.2 \%$ for pepper and $82.5 \%$ for oregano. LOQ were $5 \mu \mathrm{g} \mathrm{kg}^{-1}$ for pepper and $10 \mu \mathrm{g} \mathrm{kg}^{-1}$ for oregano. Comparing TLC - densitometry and the TLC-CCD with the photometric procedure applied in our study, it can be seen that TLC-CCD showed a better performance of the analytical method with LOQ of $1.2 \mu \mathrm{g} \mathrm{kg}^{-1}$.

Considering that TLC is the method of choice in many parts of the world, especially in developing countries, the photometric procedure developed in this study demonstrated to be a simple, efficient and low cost tool for quantitative analyses of AFB1 in peanut samples by TLC-CCD technique.

\section{ACKNOWLEDGEMENTS}

The authors thank Toxicology Laboratory at the Institute of Food Science and Technology of the Federal University of Rio Grande do Sul - UFRGS, Graduate Course of Environmental and Agriculture Microbiology - UFRGS and Coordenadoria para Aperfeiçoamento de Pessoal de Nível Superior for the graduate scholarships.

\section{REFERENCES}

1. Sherif, O. S.; Emad, E. S.; Mosaad, A. A.; Int. J. Hyg. Environ. Health 2009, 212, 347 .

2. Sulaiman, M. R.; Chye, F. Y.; Hamid, A.; Yatim, A. M.; EJEAF Che. 2007, 6, 2045.

3. Conzane, R. S.; Stenzel, W. R.; Kroh, L. W.; Dtsch. Lebensmitt. Rundsch. 2002, 98, 289.

4. Thompson, C.; Henke, S. E.; J. Wildl. Dis. 2000, 36, 172.

5. Juan, C.; Zinedine, A.; Moltó, J. C.; Idrissi, L.; Mañes, J.; Food Control 2008, 19, 849.

6. Food and Agriculture Organization of the United Nations (FAO); Food and Nutrition Papers 2004, 81, 180. 
7. Nakai, V. K.; Rocha, L. O.; Gonçalez, E.; Fonseca, H.; Ortega, E. M. M.; Corrêa, B.; Food Chem. 2008,106, 285.

8. International Agency for Research on Cancer (IARC). In IARC monographs on the evaluation of carcinogenic risks to humans, Lyon, 2002, 82.

9. Craufurd, P. Q.; Prasad, P. V. V.; Waliyar, F.; Taheri, A.; Field Crops Res. 2006, 98, 20.

10. Wang, J.; Liu, X. M.; Biomed. Environ. Sci. 2007, 20, 483.

11. Rodriguez-Amaya, D. B.; Sabino, M.; Braz. J. Microbiol. 2002, 33, 1.

12. Koe, W. J.; Arh. Hig. Rada. Toksikol. 1999, 50, 37.

13. Brasil; Diário Oficial da União, 2002, RDC n 274.

14. Sheibani, A.; Ghaziaskar, H. S.; Food Control 2009, 20, 124.

15. Leming, L.; Zhang, L. J.; Wang, P.; Wang, Y.; Chen, J.; J. Chromatogr., A 1998, 3, 815 .

16. Gilbert, J.; Anklam, E.; Trends Anal. Chem. 2002, 21, 468.

17. Lancaster, M.; Goodall, D. M.; Bergstrom, E. T.; Mccrossen, S.; Myers, P.; Anal. Chem. 2006, 78, 905.

18. Hayakawa, T.; Hirai, M.; Anal. Chem. 2003, 75, 6728.

19. Zhang, L.; Lin, X.; J. Chromatogr., A 2006, 1109, 273.

20. Liang, Y.; Baker, M. E.; Yeager, T.; Denton, M. B.; Anal. Chem. 1996, $68,3885$.

21. Prado, G.; Oliveira, M. S.; Moreira, A. P. A.; Lima, A. S.; Souza, R. A.; Alves, M. C.; Quim. Nova 2008, 31, 514.
22. Omar, S. N.; Manoj, K. M.; DheevatsaMudigere, B. E.; J. Bodywork Mov. Ther. 2007, 11, 231.

23. Moodley, K.; Murrell, H.; Comput. Geosci. 2004, 30, 609.

24. Scott, P. M.; Official Methods of Analysis of the Association of Official Analytical Chemists, Virginia, 1995, 3.

25. Aflatoxins, Official Methods of Analysis of the Association of Official Analytical Chemists, Virginia, 1995, 3.

26. Soares, L. M. V.; Rodriguez-Amaya, D. B.; J. AOAC Int. 1989, 72, 22.

27. Przybylski, W.; J. AOAC Int. 1975, 58, 163.

28. Rasband, W.; ImageJ; Image Processing and Analysis in Java; National Institute of Mental Health, USA, 1997.

29. Barnes, J.; IRAF Version 2.10; A Beginner's Guide to Using IRAF; National Optical Astronomy Observatories (NOAO), USA, 1993.

30. Sheffield, L. B.; Microsc. Microanal. 2008, 14, 898.

31. Vejchapipat, P.; Poomsawat, S.; Imvised, T.; Chongsrisawat, V.; Chittmittrapap, S.; Poovorawan, Y.; Hepatology Researc 2008, 38, 1018.

32. Smriglio, F.; Dasgupta, A. K.; Boyle, R. P.; Astronomy and Astrophysics 2006, $458,767$.

33. Sabino, M.; Milanez, T. V.; Lamardo, L. C. A.; Inomata, E. I.; Zorzetto, M. A. P.; Navas, S. A.; Stofer, M.; Rev. Microbiol. 1999, 30, 85.

34. Gnonlonfin, G. J. B.; Hell, K.; Fandohan, P.; Siame, A. B.; Int. J. Food Microb. 2008, 122, 140. 